\title{
The Sensitivity of Thermohaline Groundwater Circulation to Flow and Transport Parameters: A Numerical Study Based on Double-Diffusive Convection above a Salt Dome
}

\author{
E. Holzbecher • C. Kohfahl • M. Mazurowski • \\ A. Bącik · M. Dobies · M. Schneider
}

Received: 2 April 2009 / Accepted: 16 September 2009 / Published online: 9 October 2009

(C) The Author(s) 2009. This article is published with open access at Springerlink.com

\begin{abstract}
This article explores double-diffusive convective fluid motion in an aquifer above a salt dome. Aside from the ambient regional flow regime, the hydraulic conditions in an aquifer connected with a salt formation are determined by differences in salinity. Whereas density-driven flow patterns induced by the effect of salt have been studied before we focus on the additional effect of temperature. For the model setup, we select typical parameter values that are characteristic of not only the lowlands in Germany and Poland. For the computation of flow in a vertical cross-section, we use numerical modeling with COMSOL Multiphysics. The size and strength of eddies in the high salinity region above the aquifer base are strongly influenced by thermal effects. A sensitivity study shows a wide range of convection phenomena, ranging from the absence of convective motions via steady and oscillating circulation to unsteady fluctuating patterns. The flow and transport parameters show the highest sensitivity to the thermal Rayleigh number.
\end{abstract}

Keywords Salt dome - Thermohaline flow - Dimensionless formulation - COMSOL . Mixed convection

\section{Introduction}

The understanding of deep groundwater circulation systems in connection with salt structures is essential for a number of problems, such as nuclear waste disposal, the use of deep geothermal energy, or freshwater contamination due to ascending brines. Common

\author{
E. Holzbecher $(\varangle)$ \\ Georg-August-Universität Göttingen (GZG), Goldschmidtstr. 3, 37077 Göttingen, Germany \\ e-mail: eholzbe@gwdg.de; holzbecher@wias-berlin.de \\ C. Kohfahl · M. Schneider \\ Earth Sciences, Freie Universität Berlin (FUB), Malteserstr. 74-100, 12249 Berlin, Germany
}

M. Mazurowski · A. Bącik · M. Dobies

Pomeranian Branch, Polish Geological Institute (PGI), Wieniawskiego 20, 71-130 Szczecin, Poland 
salt structures, such as salt pillows and salt domes, can be found in almost all parts of the globe. Here, we focus on a geological setting with a salt dome, which is characteristic for the European lowlands.

Flow phenomena for fluids under the influence of salinity and temperature changes are often referred to as double-diffusive convection (Brandt and Fernando 1995). However, the different diffusivities due to mass and heat transport alone do not play a crucial role in the situation in question. Groundwater in deep sedimentary basins is often characterized by rising salinities and rising temperatures with depth. These phenomena lead to contrary effects with regard to the stability of density-stratified water. Salinity rising with depth leads to a stable configuration and predominating topography-driven flow, whereas rising temperature may produce an unstable situation with dominating buoyancy-driven flow. In the case of instability, ascending brines may contaminate upper freshwater aquifers. There is quite a low margin for acceptable salinity levels in water pumped for domestic, industrial, or public supply.

Freeze and Witherspoon (1967) stated that topography-driven flow dominates regional groundwater flow in uplifted sedimentary basins. According to Garven and Freeze (1984a) and Garven and Freeze (1984b), flow occurs where differences in elevation create differences in potential energy that drive fluid from high elevations to low elevations, developing typical maximum flow rates of 1-10 m per year in deep aquifers. Bjørlykke et al. (1988) stated that increasing salinities with depth lead to stable configurations and are not likely to be overshadowed as a consequence of decreasing densities due to higher temperatures at greater depths. They argue that this would lead to disturbed salinity profiles, which cannot be confirmed at regional scale.

Several model approaches have been developed to investigate the impact of buoyancydriven flow and topography-driven flow on the flow patterns in deep groundwater circulation systems. For simplicity, most of them consider only vertical cross-sections parallel to assumed groundwater flow directions. This two-dimensional representation is highly idealized and makes direct comparison of modeling results and field data difficult. However, the generic effects of coupling groundwater flow with heat and mass transport can be much more efficiently studied in a two-dimensional setup as it strongly reduces the significant computational demand of the corresponding fully three-dimensional setup. Early hydrogeologic models of topography-driven flow in uplifted basins focused on heat transport and neglected brine migration, due to computational limitations (Belitz and Bredehoeft 1988; Garven and Freeze 1984a,b). Other studies have focused on haline convection in the vicinity of salt domes without regarding heat transport (Evans and Nunn 1989; Evans et al. 1991; Ranganathan and Hanor 1988). The international HYDROCOIN groundwater flow modeling project was established to compare different modeling approaches for idealized flow over a salt dome (Andersson et al. 1986). A schematic picture of the modeled setting in a vertical cross-section is given in Fig 1. With reference to HYDROCOIN, Younes et al. (1999) studied the effect of molecular diffusion and compared linear and non-linear dispersion descriptions concerning haline convection.

Until, recently, very few studies have been conducted on the simultaneous solution of flow, mass, and heat transport. Oldenburg and Pruess (1998) presented simulations of thermohaline convection using the code THOUGH2 (Pruess 1991) in model systems based on the Salton Sea Geothermal System, California. They found that layered thermohaline convection is possible in hypersaline geothermal systems, provided the vertical permeability is smaller than the horizontal permeability. THOUGH2 can also be used to model thermohaline flow and transport in fractured rocks. Geiger et al. (2006) presented a new computational formulation for the simulation of transient multiphase thermohaline convection and combined the two numerical techniques finite elements and finite volumes. They focused on a novel equation 


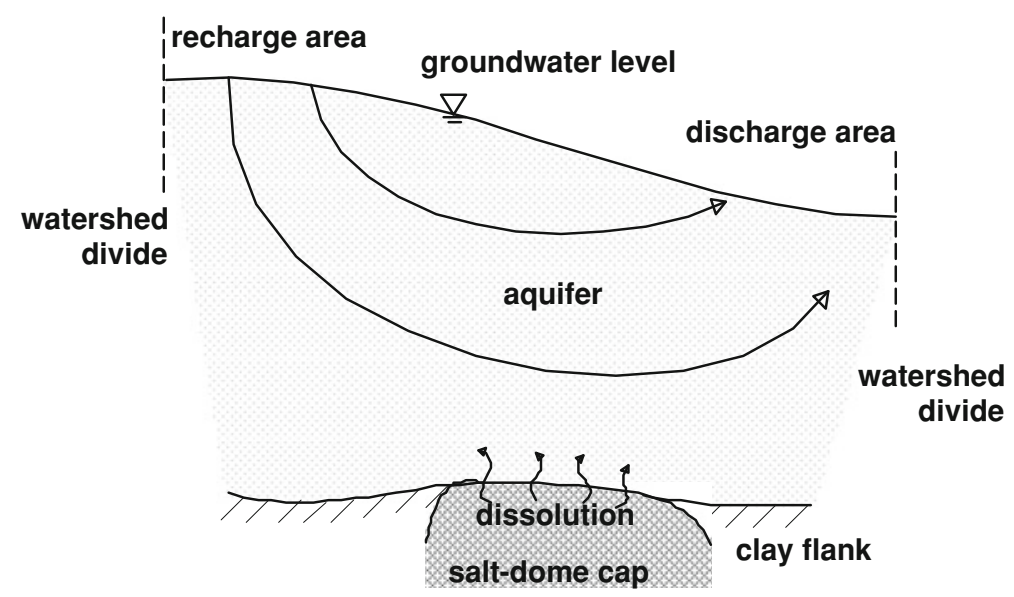

Fig. 1 Sketch of the hydrogeological situation above a salt dome

of state for the thermodynamic properties of the $\mathrm{NaCl}-\mathrm{H}_{2} \mathrm{O}$ system ranging from 0 to $750^{\circ} \mathrm{C}$ and 0-4,000 bar considering phase separations. Magrí et al. (2005) simulated thermohaline flow based on the hydrogeological setting in the NE German basin. Their results point to a situation where the subsurface fluids are generally close to convective instability, with free convection present in those regions where a sufficient, vertically continuous permeability exists. Graf and Therrien 2007 developed a numerical model that simulates dense plume migration in a chemically reactive fractured geologic material, for nonisothermal conditions. The new model is based on the existing FRAC3DVS model (Therrién and Sudicky 1996), which solves variable-saturated and multi-component transport in discretely fractured porous media. They present and compare reactive and nonreactive thermohaline flow simulations in porous and fractured media.

Although model complexity and variety have been increasing considerably in recent years, little attention has been addressed to systematic studies of parameters for non-reactive thermohaline flow simulations which are crucial for a refined understanding of strongly coupled nonlinear processes controlling deep groundwater circulation systems. This article presents a model for nonreactive thermohaline flow in porous media based on the program COMSOL Multiphysics (2006). The distinctive feature of this approach consists in the use of the streamline formulation based on dimensionless parameters, providing a steady state solution for flow and transport within a few minutes on a normal PC. This enables the performance of a large number of scenario studies to gain a refined understanding of deep fluid circulation systems, a point that has been poorly addressed in the literature until now. In order to assess the sensitivities of resulting steady state and oscillating flow patterns to selected dimensionless parameters-recalculated from real physical parameters-systematic parameter variations are presented here.

\section{Analytical Description and Numerical Model}

A conceptual model is designed for a hypothetical hydrogeological setting above a salt dome; the model is then set up and solved by COMSOL Multiphysics (2006). The model region represents a cross-section through an aquifer, which is partially connected at its base with 
a salt formation. Basic features of the model have been adopted from the conceptual model proposed in the HYDROCOIN workshop (HYDROCOIN 1988).

The main aim of the model is to identify the major parameters and processes that may cause convective flow motions. Convective flow patterns are induced by density differences, if the density changes spatially. The resulting buoyancy effect changes the entire subsurface flow regime if the density gradients are sufficiently high. As a characteristic of these motions, the vertical velocity components may rise, leading to enhanced transport from the deep subsurface to the shallow aquifer, and from there to the surface. Such a constellation is of special interest in safety assessment studies for subsurface waste repositories in salt domes.

The model is based on a formulation with three coupled differential equations, supplemented by boundary conditions. The details and the modeling software are described in the following sub-sections. A reference setup is specified, based on characteristic parameter values (Subsect. 2.3).

\subsection{Differential Equations}

Mathematical analysis of double-diffusive and thermohaline problems is based on three differential equations, which are derived from three conservation laws. The conservation of fluid mass leads to a differential equation, which is usually formulated with pressure $p$ as dependent variable:

$$
\nabla \cdot\left(\frac{\mathbf{k}}{\mu} \nabla(p+\rho g y)\right)=0
$$

with permeability tensor $\mathbf{k}$, fluid viscosity $\mu$, fluid density $\rho$ and acceleration due to gravity $g$. The coordinate $y$ denotes the vertical direction. The term in brackets is the Darcy velocity, in the following denoted by $\mathbf{v}$. Equation 1 is derived from the mass conservation principle for the fluid and from Darcy's Law (Bear 1972). Note that we omit the storage term with time derivative here, although, we are also interested in the unsteady development. Here, we take into account that the fluid is almost incompressible, so changes of fluid storage can be neglected. In the literature on density-driven flow, this simplification is mostly made with reference to the Oberbeck-Boussinesq assumption (see Holzbecher 1998a). We choose to keep viscosity as a constant, because effects of its variations can be aligned with permeability changes. In the dimensionless system used for the sensitivity study, viscosity appears in both Rayleigh numbers. As, we consider large ranges for the Rayleigh numbers, we roughly capture the effect of viscosity changes. It is well-known from studies on density-driven flow that the effect of density is usually more pronounced than the effect of viscosity, although the quantitative relative change of viscosity exceeds that of density (Holzbecher 1998a).

The conservation of salt mass leads to a transport equation with the salt concentration $c$ as dependent variable,

$$
\theta \frac{\partial c}{\partial t}=\nabla \cdot \mathbf{D} \nabla c-\mathbf{v} \cdot \nabla c
$$

and energy conservation leads to a heat transport equation with temperature $T$ as dependent variable

$$
(\rho C)_{\mathrm{fs}} \frac{\partial T}{\partial t}=\nabla \cdot \lambda \nabla T-(\rho C)_{\mathrm{f}} \mathbf{v} \cdot \nabla T
$$

(Nield and Bejan 1992; Holzbecher 1998a). Equation 2 results from the principle of mass conservation for salt and from a generalized version of Fick's Law (Bear 1972). Equation 3 is derived from the principle of energy conservation and Fourier's Law. Parameters are porosity 
$\theta$, dispersion tensor $\mathbf{D}$, Darcy velocity vector $\mathbf{v}$, the heat capacities of porous medium and fluid $(\rho C)_{\text {fs }}$ and of the fluid alone $(\rho C)_{\mathrm{f}}$, and thermal conductivity $\lambda$. With regard to heat transport, we decided to treat the isotropic situation only; for that reason, Eq. 3 contains $\lambda$ as a scalar and not as a tensor. $\lambda$ is another bulk parameter, which suffices to describe conductive heat flux, if heat dispersion is neglected. Keeping in mind the aim of a sensitivity study, we decided against using more general descriptions of flow and transport processes, as the number of parameters had to be kept small.

In the case of density-dependent flow, all three differential equations are coupled. Transport is coupled to flow via the velocity components. The feedback from transport to flow is given through the dependence of density on salt and temperature. For our numerical model, we assume a linear density dependence on both salinity and temperature. Since viscosity changes are not relevant for the geothermal gradient of the hydrogeological setting presented in this research, we neglect viscosity $(\mu)$ changes, as considered by Holzbecher (1998b). Therefore, in the temperature and salinity ranges that are measured in the vicinity of salt domes these assumptions can be justified. A further advantage of not considering viscosity changes is that we prefer to restrict the number of parameters. For that reason, we also restrict consideration of the effects of nonlinear density changes. Therefore, we concentrate on the major influence, which is the change of buoyancy.

For the following sensitivity analysis, the number of parameters required in Eqs. 1-3 is much too high. In order to limit the number of parameters, we give preference to a formulation with dimensionless variables and parameters. Such formulations have already been given in several publications, for example, by Tyvand (1980). The transition to dimensionless variables is mainly accompanied by simplifying assumptions. Most prominent is the assumption of an effective diffusivity $D_{\mathrm{s}}$ for mass transport and an effective diffusivity for heat transport, which allows the introduction of Rayleigh numbers.

For 2D setups, it is also convenient to use the streamfunction as flow variable, if the Boussinesq assumption is valid (Holzbecher 1998a). The equation for streamfunction $\Psi$ as a dependent variable is given by

$$
k_{\text {ratio }} \frac{\partial^{2} \Psi}{\partial x^{2}}+\frac{\partial^{2} \Psi}{\partial y^{2}}=\frac{R a_{\mathrm{s}}}{L e} \frac{\partial c}{\partial x}-R a_{\mathrm{t}} \frac{\partial T}{\partial x}
$$

with the solute Rayleigh number

$$
R a_{\mathrm{s}}=\frac{k \Delta \rho_{\mathrm{s}} g H}{\mu D_{\mathrm{s}}}
$$

the thermal Rayleigh number

$$
R a_{\mathrm{t}}=\frac{k \Delta \rho_{\mathrm{t}} g H}{\mu D_{\mathrm{t}}},
$$

the Lewis number

$$
L e=\frac{D_{\mathrm{t}}}{D_{\mathrm{s}}}
$$

as the ratio of the thermal and saline diffusivities $D_{\mathrm{t}}$ and $D_{\mathrm{s}}$. The ratio of porous medium permeabilities in horizontal and vertical directions is given by

$$
k_{\text {ratio }}=\frac{k}{k_{\text {vert }}}
$$

$\Delta \rho_{\mathrm{s}}$ and $\Delta \rho_{\mathrm{t}}$ denote maximum change of density due to salinity or temperature. $g$ represents acceleration due to gravity, $H$ the height of the system. Equation 4 results from the defining 
equations for the streamfunction $\Psi$ :

$$
\frac{\partial \Psi}{\partial x}=v_{\mathrm{y}} \quad \frac{\partial \Psi}{\partial y}=-v_{\mathrm{x}}
$$

and from the explicit formulation for the Darcy velocity $\mathbf{v}$ (for details see Holzbecher 1998a). The pressure terms with mixed derivatives cancel each other out, and the relatively simple right-hand side of Eq. 4 remains.

In Eq. 4 and in the following text, the variables $c$ and $T$ represent normalized salinity and temperature. Normalized variables are obtained by the transformations:

$$
x \rightarrow \frac{x}{H} \quad y \rightarrow \frac{y}{H} \quad t \rightarrow \frac{D_{t}}{H^{2}} t \quad c \rightarrow \frac{c-c_{\min }}{c_{\max }-c_{\min }} \quad T \rightarrow \frac{T-T_{\min }}{T_{\max }-T_{\min }} .
$$

The heat transport equation for normalized variables is given by:

$$
\frac{\partial T}{\partial t}=\nabla \cdot \nabla T-\gamma J(\Psi, T) .
$$

The dimensionless parameter $\gamma$ denotes the ratio of heat capacities $\rho C$ of the fluid in relation to the fluid/solid system:

$$
\gamma=\frac{(\rho C)_{\mathrm{f}}}{(\rho C)_{\mathrm{fs}}} .
$$

The Jacobian $J$-operator represents advective flux and is given by:

$$
J(\Psi, T):=v_{\mathrm{x}} \frac{\partial T}{\partial x}+v_{\mathrm{y}} \frac{\partial T}{\partial y}=\frac{\partial \Psi}{\partial y} \frac{\partial T}{\partial x}-\frac{\partial \Psi}{\partial x} \frac{\partial T}{\partial y} .
$$

Analogously, the differential equation for solute transport is given by:

$$
\theta \frac{\partial c}{\partial t}=\frac{1}{L e} \nabla \cdot \nabla c-J(\Psi, c)
$$

Altogether eight dimensionless parameters are derived from the physics of the problem: $R a_{\mathrm{s}}, R a_{\mathrm{t}}, L e, k_{\text {ratio }}, \gamma, \theta$ and the dimensionless velocity at the top of the model ( $v_{\text {bound }}$ ) and the ambient temperature at the bottom of the model $\left(T_{\mathrm{amb}}\right)$, as outlined in the next section. Two further parameters are to be added, which are connected with the geometric setup of the model as follows.

The dimensionless formulation based on the streamfunction has been used successfully for several different application cases of coupled flow and transport, such as for the classical saltwater intrusion test case defined by Henry (1960), and for the Elder problem (1967a; 1967b), for convection rolls (Holzbecher 1998a), and some others. Simms and Garven (2004) use a streamfunction formulation in non-normalized formulation. In the next section, we describe the model setup for the salt dome problem.

\subsection{Model Region and Boundary Conditions}

Within the international HYDROCOIN project (HYDROCOIN 1988), the salt dome problem was specified by Bütow (1984) and later made available to a wider public by Herbert et al. (1988). Analogously, in this study, a hypothetical vertical cross-section is laid through an aquifer, overlying the cap of a salt dome based on a hypothetical setting that can be encountered in Polish or German lowlands, according to Bujakowski et al. (2003). A sketch of the setup and boundary conditions is shown in Fig. 2, and Table 1 provides a complete detailed list of all boundary conditions. 
Fig. 2 Sketch of model region and boundary conditions for flow above a salt dome

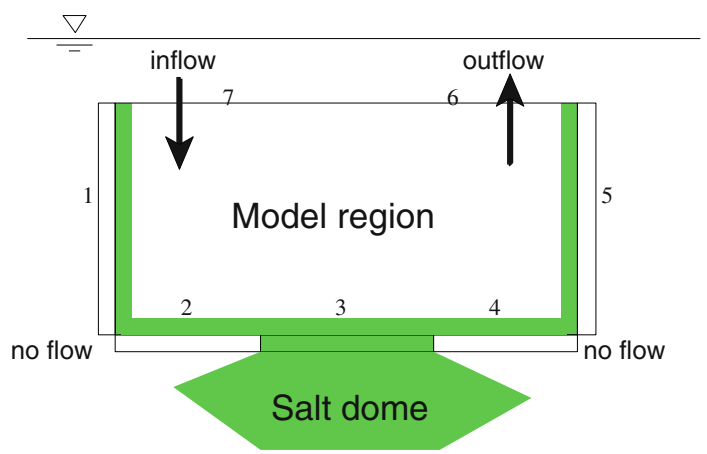

Table 1 Boundary conditions in detail; numbering of boundaries is taken from Fig. 2; variable names $\Psi$, $c$ and $T$ represent the corresponding differential equation; D indicates a Dirichlet type condition, $\mathrm{N}$ a Neumann type condition; "Values" are boundary values for the variable (in the case of D) or for the gradient of the variable (in the case of $\mathrm{N}$ )

\begin{tabular}{lllllll}
\hline Boundary & Type $\Psi$ & Value $\Psi$ & Type $c$ & Value $c$ & Type $T$ & Value $T$ \\
\hline 1.5 & $\mathrm{D}$ & 0 & $\mathrm{~N}$ & 0 & $\mathrm{~N}$ & 0 \\
2.4 & $\mathrm{D}$ & 0 & $\mathrm{~N}$ & 0 & $\mathrm{D}$ & $T_{\text {amb }}$ \\
3 & $\mathrm{D}$ & 0 & $\mathrm{D}$ & 1 & $\mathrm{D}$ & 1 \\
6 & $\mathrm{~N}$ & $v_{\text {bound }}$ & $\mathrm{N}$ & 0 & $\mathrm{~N}$ & 0 \\
7 & $\mathrm{~N}$ & $v_{\text {bound }}$ & $\mathrm{D}$ & 0 & $\mathrm{~N}$ & 0 \\
\hline
\end{tabular}

In the horizontal direction, the model region extends from one water divide to another, allowing the use of no-flow boundary conditions at the vertical boundaries. The salt dome cap is located on part of the lower edge of the cross-section, but there is no fluid exchange either between aquifer and salt dome or between aquifer and the compacted material at the vertical sides of the salt dome.

On the basis of the dimensionless streamfunction, in the model, we specify a Neumann condition for flow at the top boundary which corresponds to the specification of a constant horizontal Darcy velocity $u_{\text {bound }}$ along the top boundary. In the dimensionless formulation, the boundary velocity $v_{\text {bound }}$ is given by:

$$
v_{\text {bound }}=\frac{u_{\text {bound }} H}{D_{\mathrm{t}}}
$$

The inward and the outward fluxes, i.e., the vertical components of the velocity, at the top boundary are model output. They are calculated from the derivative of the streamfunction in horizontal direction representing the streamfunction gradients within the model and are not specified. The predefined inflow area extends from the left boundary to the first half of the top boundary, and the outflow area is represented by the second half.

The vertical boundaries are chosen at the water divides at the margin of the regional flow regime, and are implemented as no-flow boundaries. The same condition is used at the bottom of the model region, representing the base of the aquifer. We assume that the base of the aquifer lies in the same horizon as the salt dome cap, and that there is no fluid flow across the salt dome cap. In the streamfunction formulation, the no-flow condition is realized by the 
specification of a constant $\Psi$ value. That means the entire boundary is one (limit) streamline with no flux across it.

For temperature and salinity, the boundary conditions are of identical type at most parts of the model boundary. At the salt dome cap, there are Dirichlet conditions for both variables, representing a high temperature and a high salinity. As there is no fluid flow across the salt dome cap, in the model, the salt enters the system by diffusion only. At the inflow part of the top boundary, there are also Dirichlet conditions, here representing a low temperature and a low salinity of the inflowing fluid. In the terminology of normalized variables, both variables have a unit value at the salt dome and a zero value at the top boundary.

At the vertical boundaries and at the outflow part of the top boundary, we assume a no-diffusive-flux condition for both transport variables. The no-diffusive-flux condition is given by

$$
\frac{\partial T}{\partial n}=0 \quad \text { and } \quad \frac{\partial c}{\partial n}=0
$$

However, with the requirement of (16), some errors are introduced at the right-hand side of the top boundary. This is a generally accepted standard condition at outflow boundaries. In combination with the streamline Dirichlet condition at the vertical boundaries, Eq. 16 results in a no-flow condition: there is no advective and no-diffusive flux at the boundaries concerned.

The conditions for temperature and salt differ only on both sides of the salt dome, i.e., at the bottom boundary, except for the salt dome cap. There we use the Neumann condition, as given in (16) for salinity because the base rock can be assumed to be impermeable for dissolved solutes. For temperature, we use a Dirichlet condition, based on the ambient geothermal gradient in the model region. In terms of the reference parameters, we assume a temperature of $12.5^{\circ} \mathrm{C}$, which corresponds to the value of $T_{\mathrm{amb}}=0.9$ in dimensionless temperature. Temperature distributions in a heat flow model for a simple salt dome setup (which we do not report here in detail) confirm that such a value is reasonable.

\subsection{Physical and Geometrical Parameters}

\subsubsection{Physical Parameters}

Here, we discuss the parameters for the reference model setup, which are listed in Table 2. The parameter values are for a hypothetical setup, not a real salt dome. The choice of parameters was motivated by the aim to use characteristic values, most likely to be found in a real aquifer. However, there are no such typical values for some parameters. The depth of a salt dome cap, for example, is assumed to be found at a level of $100 \mathrm{~m}$ below the top of the aquifer. In reality, some salt domes reach a level of several 1,000 m depth only, while others even penetrate to the Earth's surface. We are also aware that there are regional differences in the parameter values; we based our choice on settings that can be encountered in Polish or German lowlands, and especially in the study by Bujakowski et al. (2003).

Some values in Table 2 are calculated from other parameters, such as thermal diffusivity, for which we use the formula (Häfner et al. 1992; Nield and Bejan 1992):

$$
D_{\mathrm{t}}=\frac{\lambda}{(\rho C)_{\mathrm{fs}}}
$$

Simplifying assumptions were made concerning the representation of solute diffusivity. In most models on regional transport in groundwater, a dispersion tensor is implemented 
Table 2 List of input data and intermediate parameters for the reference model

\begin{tabular}{lll}
\hline Parameter & Value $[\mathrm{unit}]$ & Source \\
\hline Height & $100[\mathrm{~m}]$ & Bujakowski et al. (2003) \\
Length & $1,600[\mathrm{~m}]$ & Assumed \\
Extension of salt dome cap & $800[\mathrm{~m}]$ & Bujakowski et al. (2003) \\
Horizontal permeability of aquifer & $1 \times 10^{-13}\left[\mathrm{~m}^{2}\right]$ & Bujakowski et al. (2003) \\
Vertical permeability & $1 \times 10^{-14}\left[\mathrm{~m}^{2}\right]$ & Assumed \\
Porosity & 0.25 & Häfner et al. (1992) \\
Dynamic viscosity of water & $1.3 \times 10^{-3}[\mathrm{~Pa} \mathrm{~s}]$ & Gavich et al. (1985) \\
Fresh water density & $999.2\left[\mathrm{~kg} / \mathrm{m}^{3}\right]$ & Bejan (1987) \\
Saline water density (max.) & $1,200\left[\mathrm{~kg} / \mathrm{m}^{3}\right]$ & Bütow (1984) \\
Minimum temperature & $8\left[{ }^{\circ} \mathrm{C}\right]$ & Bujakowski et al. (2003) \\
Maximum temperature & $13\left[{ }^{\circ} \mathrm{C}\right]$ & Bujakowski et al. (2003) \\
Ambient geothermal gradient & $2.5^{\circ} \mathrm{C} / 100\left[{ }^{\circ} \mathrm{C} / \mathrm{m}\right]$ & Bujakowski et al. (2003) \\
Density change due to temperature & $0.4\left[\mathrm{~kg} / \mathrm{m}^{3}\right]$ & Bejan (1987) \\
Specific heat capacity of water & $4,183\left[\mathrm{~J} / \mathrm{kg} /{ }^{\circ} \mathrm{C}\right]$ & (for 20 \\
Thermal conductivity of water & $0.58\left[\mathrm{~W} / \mathrm{m} /{ }^{\circ} \mathrm{C}\right]$ & (for 20 \\
Heat capacity of aquifer & $2.1 \times 10^{6}\left[\mathrm{~J} / \mathrm{m}^{3} /{ }^{\circ} \mathrm{C}\right]$ & Calculated \\
Thermal conductivity of aquifer & $0.58\left[\mathrm{~W} / \mathrm{m} /{ }^{\circ} \mathrm{C}\right]$ & Häfner et al. (1992) \\
Thermal diffusivity & $2.8 \times 10^{-7}\left[\mathrm{~m}^{2} / \mathrm{s}\right]$ & Calculated \\
Longitudinal dispersivity & $20[\mathrm{~m}]$ & Bütow (1984) \\
Transversal dispersivity & $2[\mathrm{~m}]$ & Bütow (1984) \\
Horizontal velocity at top & $3.2 \times 10^{-8}[\mathrm{~m} / \mathrm{s}]$ & Assumed \\
Solute diffusivity & $6.4 \times 10^{-8}\left[\mathrm{~m}^{2} / \mathrm{s}\right]$ & Calculated \\
\hline
\end{tabular}

(Holzbecher and Sorek 2005; Holzbecher 1998a,b). According to that approach, longitudinal and transversal dispersivities have to be introduced. Characteristic values for these, included in Table 2, were proposed by Bütow (1984) in a study for an aquifer above a salt dome in Germany. We transferred these values to a similar model based on a Fickian approach, in which the general dispersion tensor is replaced by a single (effective) solute diffusivity value. The effective diffusivity is obtained by multiplying mean velocity with mean dispersivity. For that purpose, we assumed that the mean velocity within the entire aquifer lies at a factor 5 below the maximum velocity at the upper boundary, and that the mean dispersion length is $10 \mathrm{~m}$, which lies between the values for transversal and longitudinal dispersion lengths, given by Bütow (1984). Similar assumptions were made for the salt dome test case of the HYDROCOIN project (HYDROCOIN 1988).

\subsubsection{Dimensionless Parameters}

As, we intend to utilize the dimensionless formulation introduced in Sect. 2.1, with its reduced parameter set for a sensitivity study, we compute the input values and their ranges from typical values of the original variable setup with physical parameters. In the final dimensionless model formulation, there are 10 dimensionless parameters: the length to height ratio $L / H$, 
the ratio of salt dome cap extension to the entire length $L_{0} / L$, the thermal Rayleigh number $R a_{\mathrm{t}}$, the solute Rayleigh number $R a_{\mathrm{s}}$, the Lewis number $L e$, the ratio of permeabilities in horizontal and vertical directions $k_{\text {ratio }}$, the dimensionless velocity at the top boundary $v_{\text {bound }}$, the temperature at the sides of the salt dome $T_{\mathrm{amb}}$, the ratio of heat capacities $\gamma$, and porosity $\theta$. Values for these dimensionless parameters are obtained from the set of physical parameters listed in Table 2 .

From the variables in Table 2, we obtain the following values for the dimensionless parameters:

$$
\begin{aligned}
& L / H=16, \quad L_{0} / L=0.5, \quad R a_{\mathrm{s}}=237.7, \quad R a_{t}=1.34, \quad L e=4.4 \\
& k_{\text {ratio }}=10, \quad v_{\text {bound }}=11.3, \quad \gamma=3.1, \quad \theta=0.25, \quad T_{\mathrm{amb}}=0.9
\end{aligned}
$$

Here, the boundary velocity is denoted dimensionless like all other variables. The given value corresponds to a real velocity of $1 \mathrm{~m} / \mathrm{a}$. The values for the dimensionless parameters given in (18) are the input parameters for the reference model.

\subsubsection{Parameter Ranges}

As, we also extend our numerical experiments to hydrogeological settings other than the defined reference case, we examine characteristic ranges of the real world parameters and the corresponding ranges of the dimensionless parameters. The range of observed parameter values for density, specific heat capacity, thermal conductivity, permeability, temperature, and salinity change is compiled in Table 3 . The compilations rely on parameter variability in geothermal systems, as given by Häfner et al. (1992) and in the vicinity of a salt dome (Bujakowski et al. 2003).

On the basis of the parameter ranges of Table 3, the upper and lower limits of the dimensionless parameters were calculated, and are listed in Table 4.

Table 3 Measured natural range of material parameters compiled from Häfner et al. (1992) and Bujakowski et al. (2003)

\begin{tabular}{llll}
\hline Parameter & $\min$ & $\max$ & SI Unit \\
\hline Density $(\rho)$ & 1,635 & 2,600 & $\mathrm{~kg} / \mathrm{m}^{3}$ \\
Specific heat capacity $(C)$ & 760 & 1,100 & $\mathrm{~J} /\left(\mathrm{kg}^{\circ} \mathrm{K}\right)$ \\
Thermal conductivity $(\lambda)$ & 0.42 & 7 & $\mathrm{~W} /\left(\mathrm{m}^{\circ} \mathrm{K}\right)$ \\
Permeability & $10^{-20}$ & $10^{-12}$ & {$\left[\mathrm{~m}^{2}\right]$} \\
Temperature & 8 & 150 & ${ }^{\circ} \mathrm{C}$ \\
Salinity & 0 & 200 & $\mathrm{~g} / 1$ \\
\hline
\end{tabular}

\begin{tabular}{lll}
\hline Parameter & $\min$ & $\max$ \\
\hline$R a_{\mathrm{t}}$ & 0 & 1,560 \\
$R a_{\mathrm{S}}$ & 0 & 4,800 \\
$L e$ & 4.01 & 7.27 \\
$\gamma$ & 1.02 & 3.01 \\
$v_{\text {bound }}$ & 1 & 100 \\
\hline
\end{tabular}

Table 4 Ranges of dimensionless parameters derived from Table 3 
Table 4 shows parameter ranges for the two Rayleigh numbers. The variability of several orders of magnitude for both dimensionless parameters is mainly caused by the variability of the aquifer permeability. Furthermore, the density range of water due to temperature and salinity has a major influence on the Rayleigh numbers. Also the boundary velocity defined for the model top shows a significant range. The dimensionless velocity defined in the model is derived from the groundwater flow velocity, which may vary between 0.1 and several $100 \mathrm{~m} /$ year. All the other dimensionless parameters show only minor variations.

A glance at the formulae for the dimensionless parameters, given above, reveals that some physical (non-dimensionless) parameters control several dimensionless parameters. The effect from a single parameter may thus be complex. For example, a higher permeability increases both Rayleigh numbers, which counteract each other in terms of density changes. Changes in thermal or solute diffusivities result in changes of the Lewis number and the Rayleigh number.

\subsection{Software, Numerics, and Modeling Options}

For modeling, we use the base module of COMSOL Multiphysics software, version 3.3. (2006) is a finite element software. Following the multiphysics concept of the software, we use three coupled "application modes" to model the double-diffusive system. Each of the three modes (Poisson, convection-conduction, and convection-diffusion) corresponds to a physical process or phenomenon (fluid flow, heat or energy transport, and salt transport), and to a differential equation (Eqs. 4, 9, 12). We specify the model region and the boundary conditions, as described in Subsect.2.2.

From the various discretization options, we have chosen the default quadratic Lagrange shape functions on a mesh of triangular finite elements for all variables. The reference case is solved for an irregular mesh of 3,980 mesh points and 7,486 elements, which corresponds to 46,335 degrees-of-freedom. The mesh is refined at the two edges of the top boundary (where the flow boundary condition changes), at the central position of the top boundary (where the transport boundary conditions change), and at the two outer edges of the salt dome cap at the lower boundary (where the transport boundary conditions change). For the reference case and several variations, we performed control runs using refined grids. The flow pattern on the refined mesh differed from that of the reference case only in few exceptional cases.

For most parameter variations, reported in the following, the simulation converged on the chosen mesh, using the default solver options. In the rare cases, where there was no convergence, we used grid-refinement, also automatic grid-refinement, or switched to other solver options. We also switched to transient simulations. In cases, in which neither the steady nor the transient solver developed a steady state, we assume that there is no steady state. For some parameter settings, we explored the transient simulation in addition to a direct steady solution, to find out whether steady and unsteady solutions co-exist.

\section{Results and Sensitivity Study}

The results and the sensitivity study were performed using the dimensionless formulation introduced by Eqs. 4-14. In order to describe thermohaline circulation within the natural range of physical flow parameters described above, we performed a sensitivity study using dimensionless parameter combinations which result from ranges of physical parameters. We restricted our sensitivity study to the most variable parameters, which are the thermal Rayleigh number $R a_{\mathrm{t}}$, the saline Rayleigh number $R a_{\mathrm{S}}$ and the velocity of the ambient 
groundwater $v_{\text {bound }}$. The physical meaning of these parameters can be derived from Eqs. 5, 6 , and 15 . The thermal and the saline Raleigh numbers are directly related to the groundwater's temperature and salinity gradients, respectively. The $v_{\text {bound }}$ parameter corresponds to the potential driven flow velocity of the groundwater. Therefore, the description of the sensitivity study, which is based on dimensionless parameters, can be directly related to these physical parameters also.

In order to describe the three-dimensional parameter space, we selected four values for each Rayleigh number and three values for the velocity within their parameter ranges and carried out scenarios for each combination resulting in 48 scenarios compiled in Table 5. Table 5 also reports additional simulations that were run before the sensitivity study.

Table 5 Systematic variations of dimensionless parameters. $R a_{\mathrm{t}}$ and $R a_{\mathrm{s}}$ are thermal and saline Rayleigh numbers; $v_{\text {bound }}$ is the dimensionless velocity at top boundary. $\Psi_{\min }$ and $\Psi_{\max }$ are model output values, representing global minimum and maximum of the streamfunction

\begin{tabular}{|c|c|c|c|c|c|c|}
\hline Model run & $R a_{\mathrm{t}}$ & $R a_{\mathrm{s}}$ & $v_{\text {bound }}$ & $\Psi_{\max }$ & $\Psi_{\min }$ & Characterization \\
\hline Reference model & 1.34 & 238 & 11.3 & 0.026 & -10.0 & Steady \\
\hline scen 1 & 0 & 0 & 11.3 & 0 & -10.95 & Steady \\
\hline $\operatorname{scen} 2$ & 500 & 0 & 11.3 & - & - & Fluctuating \\
\hline $\operatorname{scen} 3$ & 1,000 & 0 & 11.3 & - & - & \\
\hline scen4 & 1,500 & 0 & 11.3 & - & - & \\
\hline $\operatorname{scen} 5$ & 0 & 1,000 & 11.3 & 0.73 & -6.6 & Steady \\
\hline scen6 & 500 & 1,000 & 11.3 & 7.5 & -16.23 & Steady \\
\hline $\operatorname{scen} 7$ & 1,000 & 1,000 & 11.3 & - & - & \\
\hline scen 8 & 1,500 & 1,000 & 11.3 & - & - & \\
\hline Scen9 & 0 & 3,000 & 11.3 & & & Unrealistic \\
\hline scen9a & 0 & 2,000 & 11.3 & 1.6 & -3.40 & Steady \\
\hline scen 10 & 500 & 3,000 & 11.3 & 5.20 & -10.04 & Steady \\
\hline scen 11 & 1,000 & 3,000 & 11.3 & - & - & Fluctuating \\
\hline scen 12 & 1,500 & 3,000 & 11.3 & - & - & Fluctuating \\
\hline scen 13 & 0 & 4,800 & 11.3 & & & Unrealistic \\
\hline scen 14 & 500 & 4,800 & 11.3 & 1.91 & -4.39 & Steady \\
\hline scen 15 & 1,000 & 4,800 & 11.3 & - & - & \\
\hline scen 16 & 1,500 & 4,800 & 11.3 & & & \\
\hline scen 33 & 0 & 0 & 50 & 0 & -48.77 & Steady \\
\hline scen34 & 500 & 0 & 50 & 0 & -52.09 & Steady \\
\hline scen 35 & 1,000 & 0 & 50 & - & - & Fluctuating \\
\hline scen36 & 1,500 & 0 & 50 & - & - & Fluctuating \\
\hline scen 37 & 0 & 1,000 & 50 & 0.023 & -46.76 & Steady \\
\hline scen 38 & 500 & 1,000 & 50 & 0 & -51.06 & Steady \\
\hline $\operatorname{scen} 39$ & 1,000 & 1,000 & 50 & 0 & -54.12 & Steady \\
\hline $\operatorname{scen} 40$ & 1,500 & 1,000 & 50 & - & - & Fluctuating \\
\hline scen41 & 0 & 3,000 & 50 & 0.5 & -49.98 & Steady \\
\hline scen 42 & 500 & 3,000 & 50 & 0.27 & -48.55 & Steady \\
\hline scen 43 & 1,000 & 3,000 & 50 & 0.15 & -52.33 & Steady \\
\hline
\end{tabular}


Table 5 continued

\begin{tabular}{lllllll}
\hline Model run & $R a_{\mathrm{t}}$ & $R a_{\mathrm{s}}$ & $v_{\text {bound }}$ & $\Psi_{\max }$ & $\Psi_{\text {min }}$ & Characterization \\
\hline scen44 & 1,500 & 3,000 & 50 & - & - & \\
scen45 & 0 & 4,800 & 50 & - & - & \\
scen46 & 500 & 4,800 & 50 & 0.66 & -45.06 & Steady \\
scen47 & 1,000 & 4,800 & 50 & - & - & \\
scen48 & 1,500 & 4,800 & 50 & - & - & \\
scen49 & 0 & 0 & 100 & 0 & -96.05 & Steady \\
scen50 & 500 & 0 & 100 & 0 & -99.24 & Steady \\
scen51 & 1,000 & 0 & 100 & 0 & -101.06 & Steady \\
scen52 & 1,500 & 0 & 100 & 0 & -102.66 & Steady \\
scen53 & 0 & 1,000 & 100 & 0 & -95.95 & Steady \\
scen54 & 500 & 1,000 & 100 & 0 & -98.59 & Steady \\
scen55 & 1,000 & 1,000 & 100 & 0 & -100.52 & Steady \\
scen56 & 1,500 & 1,000 & 100 & - & - & \\
Scen57 & 0 & 3,000 & 100 & 0.11 & -92.9 & Steady \\
scen58 & 500 & 3,000 & 100 & 0.06 & -97.01 & Steady \\
scen59 & 1,000 & 3,000 & 100 & 0.03 & -99.38 & Steady \\
scen60 & 1,500 & 3,000 & 100 & 0.01 & -101.19 & Steady \\
scen61 & 0 & 4,800 & 100 & 0.33 & -89.05 & Steady \\
scen62 & 500 & 4,800 & 100 & 0.24 & -94.69 & Steady \\
scen63 & 1,000 & 4,800 & 100 & 0.17 & -98.14 & Steady \\
scen64 & 1,500 & 4,800 & 100 & 0.69 & -100.11 & Steady \\
scen65 & 10 & 238 & 11.3 & 0.02 & -10.0 & Steady \\
scen66 & 200 & 238 & 11.3 & 0 & -17.0 & Steady \\
scen67 & 210 & 238 & 11.3 & 0 & -13.77 & Steady and oscillating \\
scen68 & 400 & 238 & 11.3 & 0 & -17.2 & Oscillating \\
scen69 & 425 & 238 & 11.3 & 5.3 & -18.0 & Oscillating \\
scen70 & 500 & 238 & 11.3 & 5.4 & -22.0 & Oscillating \\
\hline & & & & & &
\end{tabular}

Table 5 gives the global minimum and maximum of the streamfunction $\Psi_{\min }$ and $\Psi_{\max }$ for each model run, as these are significant quantities for the characterization of the flow field. Two scenarios (scenario 9 and scenario 13) reveal a steady state flow field dominated by eddies leading to inflow across the right part of the top boundary, which is not considered in the boundary conditions. Therefore, the results of these two scenarios are not realistic, and are not documented further. In scenario 9, the saline Rayleigh number has been altered to 2,000 (scenario 9a).

The presented cross-sections of flow fields show flowlines represented by streamfunction contours, arrows representing velocities, filled contours for concentration, and the maximum and minimum value of the streamfunction in the model domain.

Owing to the formulation of the streamline function, negative (positive) values of $\Psi$ indicate counterclockwise (clockwise) water circulation, respectively. $\Psi_{\min }$ represents the mass of fluid in a counterclockwise circulating eddy, here represented by the potential driven flow forming the lower half of an eddy. Analogously, $\Psi_{\max }$ represents the mass of fluid in 


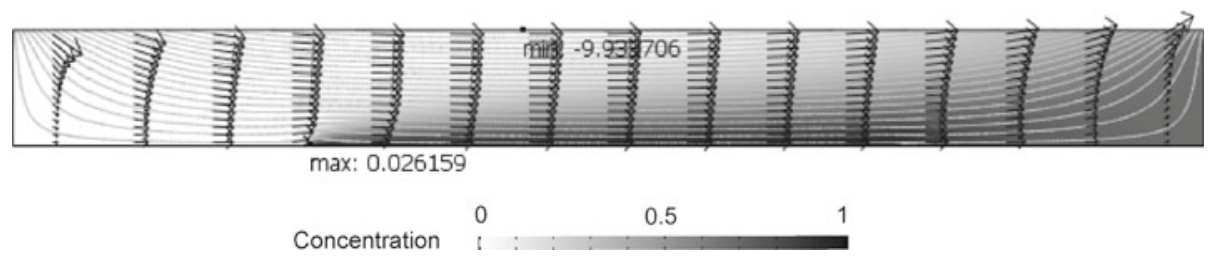

Fig. 3 Calculated flow field for the reference case. Surface color for normalized salinity. Lines represent equidistant streamlines, and arrows are flow velocities. $x$-axis and $y$-axis represent length units. Vertical exaggeration is 2

a clockwise circulating cell driven by buoyancy, if such an eddy appears. Thus low values of streamfunction can be observed when there are no eddies at all, i.e., if there is no change of flow direction at the bottom of the model region. If eddies appear, the situation is more complex. Eddies with alterating circulation correspond to positive and negative values of the streamfunction. However, it is not possible that a single eddy with negative $\Psi$ appears. Thus in the case of dominating buoyancy effects with the appearance of several strong eddies, there are peak high (positive) and low (negative) values of the streamfunction. These general characteristics of the flow pattern enable an interpretation of the flow pattern, based on $\Psi_{\min }$ and $\Psi_{\max }$.

\subsection{Reference Case}

The result of the reference case is shown in Fig. 3. The visualization shows that the potential flow field is almost undisturbed by any density effect. Although no eddy is visible in the figure, the positive maximum of 0.026 for $\Psi$ at the bottom of the model region indicates a locally re-circulating eddy. The fluid circulating in the eddy is approximately 2.5 per mille of the ambient flow above (the maximum of streamfunction is $\approx 10$ ). A zoom into the streamline pattern at the front of the salt dome confirms that there is some local re-circulation.

If the boundary condition with fixed concentration is specified at the entire bottom of the model, i.e., if the salt dome cap of the reference situation is extended, the re-circulating eddy disappears. From this comparison, we conclude that the emergence of the eddy is related to horizontal density differences in the vicinity of the contact between the diapir and the adjacent rock with non-mineralized water. High salinity brine originating from dissolution of salt at the salt dome cap moves opposite to the main flow direction along the base of the aquifer. The local situation at the upstream margin of the salt dome cap is identical to that of saltwater intrusion (Henry 1960; Holzbecher 1998a), and the resulting local flow patterns can be explained the same way.

We compared the flow pattern of the reference case with the output from a run, in which the thermal regime was completely neglected (by setting the thermal Rayleigh number to 0 ). The results of both runs differ only marginally: the maximum of the streamfunction is slightly higher in the variation. We conclude that the effect of temperature is marginal for the reference situation. In the following, we examine the combinations of dimensionless parameters for which the thermal regime becomes relevant.

\subsection{Steady-State Model Runs}

We perform a sensitivity study, changing the five most important dimensionless parameters systematically within the range given in Table 3 . For most steady state scenarios, we find a 


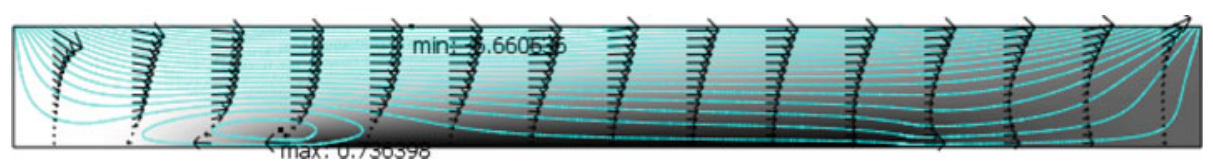

Fig. 4 Calculated flow field for reduced thermal influence and increased saline influence (scen5: $R a_{\mathrm{t}}=$ $0, R a_{\mathrm{s}}=1,000, v_{\text {bound }}=11.3$ )

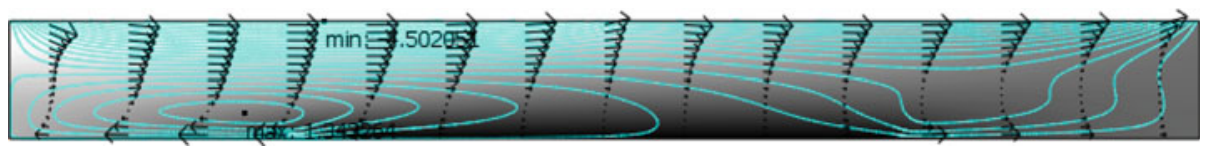

Fig. 5 Calculated flow field for highly increased saline influence (scen9a: $R a_{\mathrm{t}}=0, R a_{\mathrm{s}}=2,000, v_{\text {bound }}=$ $11.3)$

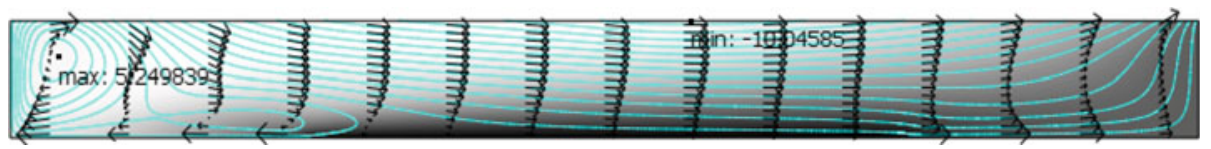

Fig. 6 Calculated flow field for increased thermal influence (scen10: $R a_{\mathrm{t}}=500, R a_{\mathrm{s}}=3,000, v_{\text {bound }}=$ 11.3)

convection solution with one or more eddies. The formation of eddies as an effect of salinity differences (i.e., elevated $R a_{\mathrm{s}}$ ) occurs at the left salt dome edge. The eddy formation is related to horizontal density differences due to the increased concentration gradients at the diapir edge-as described for the reference model in the previous sub-section, and as depicted in Figs. 3 and 4.

The flow pattern of Fig. 4 is obtained if the thermal regime becomes weaker, represented by decreased $R a_{\mathrm{t}}$, and the saline regime becomes stronger, represented by an increased $R a_{\mathrm{S}}$ (scen5, see Table 5). The eddy on the left model margin was induced by the saline regime, and is clearly visible in the figure.

A further increase of the saline Rayleigh number to 2,000 leads to the formation of a further eddy-like flow pattern above the right edge of the salt dome, as shown in Fig. 5 (scen9a). In fact, the fluid does not perform a complete circulation: only the velocity in vertical direction is increased, downward at an upstream position and upward at a downstream position. A stagnation point emerges in the solution. The half-eddy or three-quarter eddy can again be attributed to horizontal salinity gradients: concentration levels above the salt dome are kept high owing to the permanent dissolution of salt. On the other hand, owing to the permanent supply of fresh water, salinity levels are lower within the reach of the potential flow regime, not only near the upper boundary but also near the right boundary.

For the dominant saline regime, the locations of eddies are almost fixed for the different scenarios: there is one eddy upstream from the salt dome edge, and a half-eddy above the downstream edge of the salt dome. By contrast, the location of eddies due to an increased $R a_{\mathrm{t}}$ is variable and depends on the specific parameter combination of the scenario. Figure 6 shows the formation of two eddies in scenario 10 (see Table 5), in which the saline and thermal Rayleigh numbers are increased. The eddy in the upper part on the left can be attributed to the thermal regime, whereas the eddy at the base near the edge of the salt dome is due to the saline regime. 


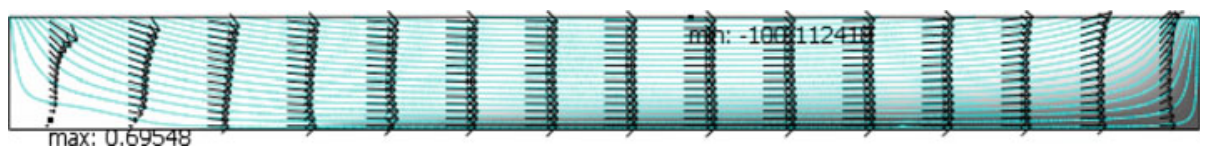

Fig. 7 Calculated flow field for an increased potential velocity (scen64: $R a_{\mathrm{t}}=1,500, R a_{\mathrm{S}}=$ $\left.4,800, v_{\text {bound }}=100\right)$

As the thermal and the saline Rayleigh numbers influence the fluid density in an opposite manner, they also counteract each other with regard to the formation of eddies. This can be observed by comparing Figs. 5 and 6 . The elevation of the thermal Rayleigh number in Fig. 6 leads to the disappearance or weakening of the second eddy on the right edge of the salt dome visible in Fig. 5. This is also supported by comparing $\Psi_{\max }$ values of scenarios showing similar flow patterns such as scenarios 37-39: $\Psi_{\max }$ values decrease with rising thermal Rayleigh numbers. Eddies, originating from a strong saline regime, are weakened by the thermal regime.

Increasing the boundary velocity leads to smaller values of $\Psi_{\max }$ (representing the buoyancy influence of salinity and temperature) and more negative values of $\Psi_{\min }$ (representing potential flow). A stronger potential flow reduces the tendency of eddy formation. Figure 7 shows a potential driven flow field simulated in scenario 64 in spite of a very high Rayleigh number, which is due to the high flow velocity of 100. In contrast to Fig. 3 of the reference model, which also shows mainly a potential driven flow field, no upwelling of the flowlines is visible at the left edge of the salt dome. The zone of elevated salinity is restricted to a narrow stretch in the vicinity of the bottom, and of the right boundary due to the higher dominance of advective flow.

In conclusion, these scenario calculations revealed two types of steady state solutions: (i) a mainly potential driven flow field and (ii) steady state solutions with eddies. Most solutions fall into one of these two categories. Scenario 56 can be understood as a transition situation between these two steady state solutions turning from a nearby type (i) situation (scen52) to a nearby type (ii) situation (scen60). The transitory position between the two steady flow fields may be the reason that a steady state solution could not be found in scenario 56.

The scenario calculations revealed that for certain input data the direct modeling approach did not converge to a steady state. Particularly, high $R a_{\mathrm{t}}$ constrict the existence of steady state solutions, especially in combination with low $R a_{\mathrm{s}}$ and low $v_{\text {bound }}$. The critical values in which no steady state solution exists depend on the parameter combination. Salinity gradients expressed by saline Rayleigh numbers and high flow velocities expressed as $v_{\text {bound }}$ velocity destabilize eddies formed by thermal Rayleigh numbers and lead to steady state solutions also for higher thermal numbers. Table 5 shows the region in the three-dimensional parameter space in which steady state solutions exist: it is below thermal Rayleigh numbers of 500-1,500 in combination with high saline Rayleigh numbers and high flow velocities.

\subsection{Transient Simulations}

Transient simulations were performed for selected situations investigating the range of obtained solutions focusing on parameter combinations at the limit of existing solutions obtained by steady state options. Furthermore, transient simulations were performed for parameter combinations without a solution using the steady state mode. 


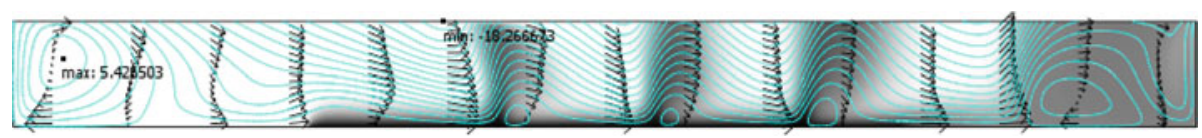

Fig. 8 Calculated transient flow field for an increased thermal Rayleigh number of 425 (scenario 69). Lines represent equidistant streamlines and black arrows are temporary flow velocities; dimensionless simulation time: 52

From the simulations, we obtain three types of solutions: steady state, oscillating, or fluctuating. After an initial settling time, some transient simulations strictly follow an oscillatory pattern. If the temporal behavior of one of the state variables is investigated at a selected position within the model region, a periodic change between a maximum and a minimum is observed. We call this oscillating, whereas we use the more general term "fluctuating" also for non-oscillatory transient regimes.

We demonstrate the behavior of the solutions for a set of scenarios, in which $R a_{\mathrm{t}}$ is increased from the reference case, as the only parameter. The steady state solver yields steady state solutions up to $R a_{\mathrm{t}} \approx 395$. For thermal Rayleigh numbers slightly higher than this value no solution was obtained, even with locally or globally refined meshes. In analogy to classical Benard convection in porous media (Holzbecher 1998a,b), this may be called the critical Rayleigh number. Here, the value is valid only for the reference salt dome situation, as defined above.

Using the transient modeling option for the same parameter setting, we observed an oscillating flow pattern. The dependence of the oscillating solution on initial variable distribution was examined, using a convection or a potential flow field as initial condition. After the settling time the oscillating solution was identical in both scenarios. The flow pattern in Fig. 8 shows a snapshot (at dimensionless time $t=1$ ) from the simulation for a thermal Rayleigh number of 425 (scen69).

A strong eddy, reaching from the top to the bottom of the aquifer, emerges near the inlet. At the center of the eddy, the streamfunction reaches its global maximum. Moreover, at the bottom of the model region, five eddies can be identified in Fig. 8. The wavelike pattern of the overlying flow corresponds to eddies in the lower part of the cross-section. In the simulation, eddies move from left to right, form at the front edge of the salt dome, grow and move downstream. All this happens periodically with a constant time period. The oscillating behavior can be observed at most positions in the model region. As an example, in Fig. 9, the oscillations of the streamfunction are shown at the center of the eddy adjacent to the left boundary, where $\Psi$ reaches its maximum. The maximum value of the streamfunction corresponds to the mass or volume circulating in the eddy.

Figure 9 shows clearly that, after an initial settling time, the solution strictly follows an oscillatory pattern. As already stated, the oscillations are connected with the emergence, strengthening and migration of eddies at the upstream salt dome edge.

For further elevated thermal Rayleigh numbers, the flow regime takes a more irregular pattern. Figures 10 and 11 show snapshots from the transient development of the oscillating flow pattern for $R a_{\mathrm{t}}=425$ (scen69). Together with Fig. 8, these figures visualize the origin of the oscillations at the locations within the flow field. Small eddies appear at the left margin of the salt dome cap. While growing, they move to the right and finally disappear at the right side of the model region.

We started several model runs with the transient option for thermal Rayleigh numbers $R a_{\mathrm{t}}<400$. It turns out that the critical Rayleigh number of 395, found above for the transient to the oscillatory state, is not recognized by the transient simulations if a transient state from the former simulation is used as initial value. The numerical solutions remain oscilla- 


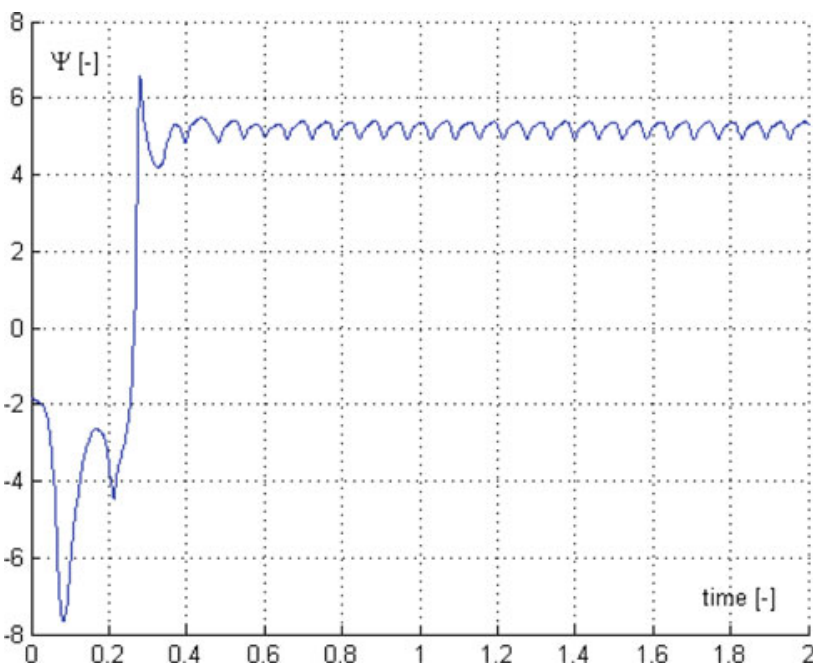

Fig. 9 Temporal change of streamfunction $\Psi$ at the center of the upstream eddy for scenario 69 (see Fig. 8); reveals oscillatory behavior of the flow pattern

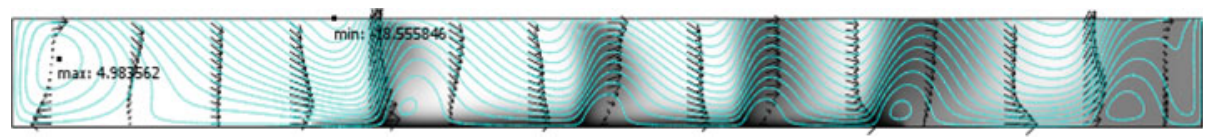

Fig. 10 Calculated transient flow field for an increased thermal Rayleigh number of 425 (scenario 69); dimensionless simulation time: 55

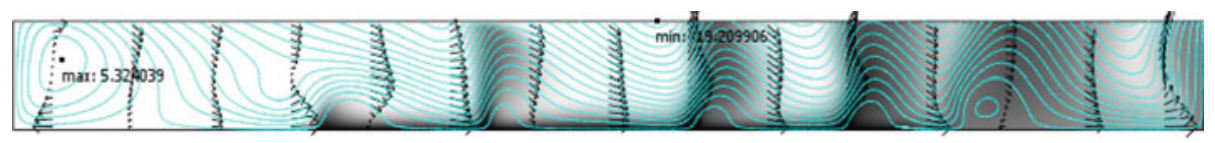

Fig. 11 Calculated transient flow field for an increased thermal Rayleigh number of 425 (scenario 69); dimensionless simulation time: 58.5

tory. With further reduced $R a_{\mathrm{t}}$ values, eddies become smaller and weaker. Finally, for $R a_{\mathrm{t}}$ values between 200 and 210 oscillations disappear and give place to the steady state solution.

Summarizing, we state that all transient simulations of this demonstration with Rayleigh numbers higher than 210 show oscillating flow patterns. Steady-state and transient solutions co-exist for Rayleigh numbers between 210 and 395. For other parameter combinations, oscillatory regimes were observed, too. For some other scenarios, periodic oscillations were not observed. We distinguish these two cases in the last column of Table 5, where the transient flow regime is characterized as "oscillatory" or "fluctuating" (if there are no oscillations). For scenarios in which transient and steady state solutions were found, this is also indicated. However, not all possible parameter combinations are explored by both model options.

\section{Conclusion}

We examined the effect of temperature on flow patterns in an aquifer above a salt dome cap. For a reference situation with parameters typical for the European lowlands, we found that 
the effect of the geothermal regime on the flow pattern is marginal. For a typical setting, the temperature gradients are not sufficient to destabilize the flow pattern, which is mainly shaped by the interaction of the hydraulic potential- and salinity-induced density-driven flow.

By systematically varying the relevant parameters of the reference case, we found different flow patterns: steady state, as well as oscillating and fluctuating solutions. Patterns of partially or completely circulating flow emerge mainly due to salinity-induced density differences in a horizontal direction. At the two marginal locations of the salt dome at the bottom boundary of the model region, flow instabilities appear that can influence the entire flow pattern. At the downstream edge of the salt dome, horizontal density differences mostly decrease toward the right horizontal boundary and toward the outlet. By contrast, the front edge of the diapir remains the most prominent location for the emergence of eddies. The reason for the different behavior on both sides of the salt dome cap is that at the latter location dispersive/diffusive fluxes and advective fluxes have opposite directions, whereas at the downstream edge their direction is the same.

Of the dimensionless parameters studied, the Lewis number and heat capacity ratio are of minor importance, as their maximum variation, with factors of 2 and 3, is relatively low. By contrast, the salt Rayleigh number shows a high sensitivity for data ranges that are of interest in real applications. High Rayleigh numbers stand for high permeability, high density difference, high aquifer thickness, low diffusivity and low viscosity, or for combinations of these.

The greatest variety of flow patterns within the examined parameter range is due to changes of the thermal Rayleigh number. The above-mentioned flow instability at the front edge of the diapir induces different types of eddies. For low values of $R a_{\mathrm{t}}$ one stable eddy emerges. For increased values further stable eddies appear, which start to move if $R a_{\mathrm{t}}$ is increased further. A regular pattern with oscillations finally turns into a fluctuating regime. For some parameter settings, we obtained solutions for direct steady state and transient simulations.

The sensitivity study for the most relevant dimensionless parameters yielded the highest sensitivities for (i) the thermal Rayleigh number, (ii) the salt Rayleigh number, and (iii) the velocity at the top, representing ambient potential flow. Thus it can be concluded that there is a high sensitivity to all parameters appearing in Eqs. 5 and 6 and to all parameters affecting potential flow. The area of the three-dimensional parameter space spanned by $R a_{\mathrm{t}}, R a_{\mathrm{s}}, v_{\text {bound }}$, in which steady state solutions exist, varies between thermal Rayleigh numbers of 500-1,500 in combination with high saline Rayleigh numbers and high flow velocities. We conclude that highly permeable geological formations, elevated geothermal gradients in combination with weak groundwater flow, constitute the most suitable conditions for the emergence of thermally induced convective flow patterns. This means that the influence of the thermal regime becomes relevant only for a weak regime of the ambient flow, i.e., if velocities at the top boundary are lower than $0.1 \mathrm{~m} / \mathrm{a}$. Such a condition is more likely in deep aquifers, where velocities are usually smaller than in aquifers influenced by the relatively strong near-surface hydraulic potential.

Acknowledgements The authors are grateful to Morphotectonic Map of the European Lowland Area (MELA), a project funded by the European Community.

Open Access This article is distributed under the terms of the Creative Commons Attribution Noncommercial License which permits any noncommercial use, distribution, and reproduction in any medium, provided the original author(s) and source are credited. 


\section{References}

Andersson, K., Grundfeld, G., Hodgkinson, D.P., Lindbom, B., Jackson, C.P.: HYDROCOIN level 1 final report: verification of groundwater flow models. Swedish Nuclear Power Inspectorate (SKI), Stockholm (1986)

Bear, J.: Flow Through Porous Media. Elsevier, New York (1972)

Bejan, A.: Convective heat transfer in porous media. In: Kakaç, S., Shah, R.K., Aung, W. (eds.) Handbook of Single-Phase Convective Heat Transfer, Chap. 16, Wiley, New York (1987)

Belitz, K., Bredehoeft, J.: Hydrodynamics of Denver basin: explanation of subnormal fluid pressures. Am. Assoc. Petrol. Geol. Bull. 72, 1334-1359 (1988)

Bjørlykke, K., Mo, A., Palm, E.: Modeling of thermal convection in sedimentary basins and its relevance to diagenetic reactions. Mar. Petrol. Geol. 5, 338-351 (1988)

Brandt, A., Fernando, H.J.S. (eds.): Double-diffusive Convection. Am. Geophys. Union, Geophys. Monograph 94 (1995)

Bujakowski, W., Czerwinski, T., Garlicki, A., Jarzyna, J., Mularz, S., Tarkowski, R.: Termiczna Characterystyka Górotworu w Rejonie Wysadów Solnych. Polska Akademia Nauk, Wydawnictwo IGSMiE PAN, Kraków, p. 136 (2003, in Polish with English abstract)

Bütow, E.: Salt Water Distribution in a Saturated Porous Medium, Proposal for a Test Problem HYDROCOIN Level 1 Case 5. HYDROCOIN, Stockholm (1984)

COMSOL Multiphysics, Version 3.3: COMSOL AB, Tegnérgatan 23, SE-111 40 Stockholm, Sweden (2006)

Elder, J.W.: Steady free convection in a porous medium heated from below. J. Fluid Mech. 27, 29-48 (1967a)

Elder, J.W.: Transient convection in a porous medium. J. Fluid Mech. 27(3), 609-623 (1967b)

Evans, D., Nunn, J.: Free thermohaline convection in sediments surrounding a salt column. J. Geophys. Res. 94(B9), 12413-12422 (1989)

Evans, D., Nunn, J., Hanor, J.: Mechanisms driving groundwater flow near salt domes. Geophys. Res. Lett. 18, 927-930 (1991)

Freeze, R., Witherspoon, P.: Theoretical analysis of regional groundwater flow. 2. Effect of water-table configuration and subsurface permeability variation. Water Resour. Res. 3, 632-634 (1967)

Garven, G., Freeze, R.: Theoretical analysis of the role of groundwater flow in the genesis of stratabound ore deposits: 1. Mathematical and numerical model. Am. J. Sci. 284, 1085-1124 (1984a)

Garven, G., Freeze, R.: Theoretical analysis of the role of groundwater flow in the genesis of stratabound ore deposits: 2. Quantitative results. Am. J. Sci. 284, 1125-1174 (1984b)

Gavich, I.K., Lucheva, A.A., Semionova-Erofeeva, S.M.: Sbornik zadach po obscej gidro-geologii, Moskva Nedra (1985, in Russian)

Geiger, S., Driesner, T., Heinrich, C.A., Mattäi, S.K.: Multiphase thermohaline convection in the earth's crust: I- a new finite element-finite volume solution technique combined with a new equation of state for $\mathrm{NaCl}-\mathrm{H}_{2} \mathrm{O}$. Transp. Porous Med. 63, 399-434 (2006)

Graf, T., Therrien, R.: Coupled thermohaline groundwater flow and single-species reactive solute transport in fractured porous media. Adv. Water Resour. 30, 742-771 (2007)

Häfner, F., Sames, D., Voigt, H.-D.: Wärme- und Stofftransport. Springer, Berlin (1992, in German)

Henry, H.R.: Salt Intrusion into Coastal Aquifers. Int. Assoc. Sci. Hydrol. Publ. 52, 478-487 (1960)

Herbert, A.W., Jackson, C.P., Lever, D.A.: Coupled groundwater flow and solute transport with fluid density strongly dependent upon concentration. Water Resour. Res. 24, 1781-1795 (1988)

Holzbecher, E.: Modeling Density-Driven Flow in Porous Media. Springer, Heidelberg (1998a)

Holzbecher, E.: The influence of variable viscosity on thermal convection in porous media. In: Nowak, A.J., Brebbia, C.A., Bialoki, R., Zerroukat, M. (eds.) Proceedings of the Heat Transfer 98, pp. 115-124. Computational Mechanics Publications, Southampton (1998b)

Holzbecher, E., Sorek, S.: Numerical models of groundwater flow and transport. In: Anderson, M. (ed.) Encyclopaedia of Hydrological Sciences, Vol. 4, Part 13, Art. 155, pp. 2401-2414. Wiley, Chichester (2005)

HYDROCOIN: Groundwater Hydrology Modelling Strategies for Performance Assessment of Nuclear Waste Disposal, Level 1: Code Verification. OECD, Paris (1988)

Magrí, F., Bayer, U., Clausnitzer, V., Jahnke, C., Fuhrmann, J., Möller, P., Pekdeger, A., Tesmer, M., Voigt, H.: Deep reaching fluid flow close to convective instability in the NE German basin-results from water chemistry and numerical modelling. Tectonophysics 397, 5-20 (2005)

Nield, D.A., Bejan, A.: Convection in Porous Media. Springer, New York (1992)

Oldenburg, C.M., Pruess, K.: Layered thermohaline convection in hypersaline geothermal systems. Transp. Porous Med. 33, 29-63 (1998)

Pruess, K.: TOUGH2: A General Purpose Numerical Simulator for Multiphase Fluid and Heat Flow, Report No. LBL-29400. Lawrence Berkeley Laboratory, Berkeley, CA (1991) 
Ranganathan, V., Hanor, J.S.: Density-driven groundwater flow near salt domes. Chem. Geol. 74, 173188 (1988)

Simms, M.A., Garven, G.: Thermal convection in faulted extensional sedimentary basins: theoretical results from finite element modelling. Geofluids 4, 109-130 (2004)

Therrién, R., Sudicky, E.: Three-dimensional analysis of variably saturated flow and solute transport in discretely-fractured porous media. J. Contam. Hydrol. 23(6), 1-44 (1996)

Tyvand, P.A.: Thermohaline instability in anisotropic porous media. Water Resour. Res. 16, 325-330 (1980)

Younes, A., Ackerer, P., Mose, R.: Modeling variable density flow and solute transport in porous medium: 2. Re-evaluation of the salt dome flow problem. Transp. Porous Med. 35, 375-394 (1999) 